\title{
The acoustic waves propagation in a cylindrical wave- guide with the laminar flow
}

\author{
Alexander Petrov ${ }^{1, *}$ and Valentina Rumyantseva ${ }^{2, * *}$ \\ ${ }^{1}$ MISIS National University of Science and Technology \\ ${ }^{2}$ Bauman Moscow State Technical University
}

\begin{abstract}
The problem of modeling of acoustic wave propagation in inhomogeneous flow is considered. There is an approximate analytical solution of the hydrodynamics equations in the presence of annular acoustic oscillations source in the case of laminar flow.

Special attention is to paid to the propagation of acoustic waves modes. The amplitudes and phases dependences of the individual modes on the Mach number in the linear approximation were established.
\end{abstract}

\section{Introduction}

The task of the gas-air flows measurement is relevant for many areas of science and technology, for example, such as medicine (spirometry), occupational safety, ventilation control, etc.

A number of requirements to the sensors of air flow speeds, are imposed, such as: accuracy, reliability, inertia-free, large dynamic range, relatively low cost. The ability to meet these requirements depends on the measurement principle. In our opinion, the most promising method for measuring of the velocity of gas-air flow is the acoustic phase method proposed by Professor Shkundin (Russia) [1].

The method consists of mounting two piezoelectric ring transducers (main parts of the sensor) into the cylindrical waveguide-air duc's wall . One of them is an acoustic wave transmitter and the other is a receiver. Periodically they turn their roles. An external flow creates a flow within the sensor as well. The phase of the acoustic signal depends on the flow rate in the waveguide-air duct. The receiver and transmitter change roles at each measurement step. Flow velocity values are determined by the phase difference between the signals propagating along and against the flow.

Samples of such sensors already exist and successfully operate as part of mine anemometers. A theoretical description of this method was developed. However, all models of aeroacoustic interaction use the approximation of uniform flow. This approximation is correct in the case of large Reynolds numbers, when the average velocity plot is similar to rectangular.

In this paper, we consider the description of the aeroacoustic interaction with a laminar flow, i.e. a flow with a parabolic velocity plot. To improve the existing devices, as well

\footnotetext{
*e-mail: petrovipmech@gmail.com

**e-mail: valar@bmstu.ru
} 
as to develop new ones: spirometers, anemometers, flowmeters, it is necessary to theoretically describe the physical processes underlying the measurement principle, i.e. the model of aeroacoustic interaction in the waveguide-air duct.

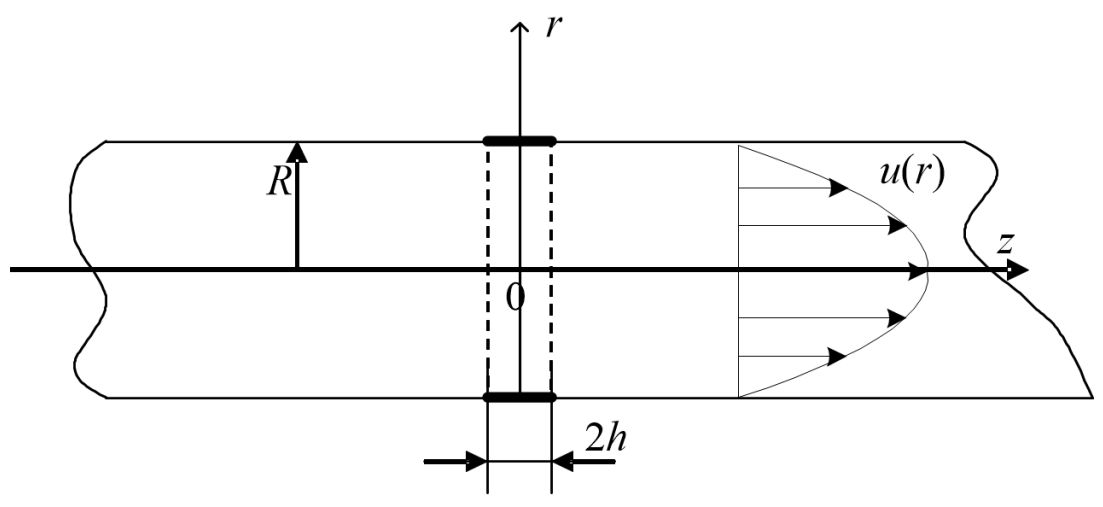

Figure 1. Cilindrical waveguard

\section{Problem statement}

Consider an acoustic waveguide-air duct with a source of acoustic vibrations having the form of a ring mounted in the wall of the waveguide. We obtain the acoustic field of the wave propagating from the source. The ring oscillates in the radial direction according to the harmonic law with a frequency of $\omega$. Since we will not consider reflections from the ends of the waveguide, let us assume that in this approximation the waveguide of unlimited length is considered. The walls of the waveguide are considered infinitely rigid. We introduce a cylindrical coordinate system, as shown in Fig. 1. The problem has cylindrical symmetry, so vectors have only two components: $\mathbf{r}=(r, z)$. Let a laminar flow with a velocity profile constant along the $z$ axis be established in the waveguide. Then, according to [2], its field will be determined by the expression (1).

$$
u(r)=2 \bar{u}\left(1-\frac{r^{2}}{R^{2}}\right)
$$

where $\bar{u}$-the average in cross-section velocity.

To describe the wave field, we use the acoustic approximation. We believe that the parameters of the medium are the sum of constant and small oscillatory component. $v_{z}=$ $u(r)+v_{z}^{\prime}, v_{r}=v_{r}^{\prime}, \rho=\rho_{0}+\rho^{\prime}, p=p_{0}+p^{\prime}$, where $\mathbf{v}=\left(v_{r}, v_{z}\right)=\mathbf{v}(\mathbf{r}, t), \rho=\rho(\mathbf{R}, t)$, $p=p(\mathbf{r}, t)$ - speed, density and pressure of the medium, respectively. The boundary condition for the velocity on the inner wall of the waveguide will be:

$$
\left.v_{r}\right|_{r=R}=\left\{\begin{array}{c}
V_{0} e^{\mathrm{i} \omega t},|z| \leq h / 2 \\
0,|z|>h / 2
\end{array}\right.
$$

Here $V_{0}$ is the surface of the ring vibration speed amplitude, $\omega$ is the angular frequency of oscillation, $\mathrm{i}$ is the imaginary unit.

We solve the basic system of hydrodynamics [3,5-9]. For the air in the acoustic approximation the viscosity is small. Therefore, the equation of motion for us will be the Euler 
equation for the ideal gas. The equation of continuity:

$$
\frac{\partial \rho}{\partial t}+\frac{\partial}{\partial z}\left(\rho v_{z}\right)+\frac{\partial}{\partial r}\left(\rho v_{r}\right)+\frac{\rho v_{r}}{r}=0
$$

The equations of motion (longitudinal and radial projections) are

$$
\begin{aligned}
& \frac{\partial v_{z}}{\partial t}+v_{z} \frac{\partial v_{z}}{\partial z}+v_{r} \frac{\partial v_{z}}{\partial r}=-\frac{\partial p}{\rho \partial z} \\
& \frac{\partial v_{r}}{\partial t}+v_{z} \frac{\partial v_{r}}{\partial z}+v_{r} \frac{\partial v_{r}}{\partial r}=-\frac{\partial p}{\rho \partial r}
\end{aligned}
$$

where $\triangle=\frac{\partial^{2}}{\partial r^{2}}+\frac{1}{r} \frac{\partial}{\partial r}+\frac{\partial^{2}}{\partial z^{2}}$

However, the inhomogeneity of the flow does not make the velocity field potential (viscosity is taken into account when calculating the shape of the velocity plot), so the solution will be expressed in the form of the acoustic potential $\Phi$ and the current function $\Psi$. The vibration speed dependence on these functions has the form:

$$
v_{z}^{\prime}=\frac{\partial \Phi}{\partial z}+\frac{1}{r} \frac{\partial \Psi}{\partial r}, v_{r}^{\prime}=\frac{\partial \Phi}{\partial r}+\frac{1}{r} \frac{\partial \Psi}{\partial z}
$$

\section{Problem solution}

\subsection{System of equations obtaining}

We obtain a system of differential equations with respect to potentials and acoustic pressure. To do this we substitute the expression of the medium parameters into the main system and apply linear approximation for acoustic (vibrational) components. We assume that sound propagation is adiabatic, supplementing the system with $p=c^{2} \rho$, where $c^{2}=\frac{\partial p^{\prime}}{\partial \rho}, c$ is the adiabatic speed of sound.

In this case, we introduce the auxiliary function $F$, and also represent the density as a relative dimensionless value: $\widetilde{\rho}=\frac{\rho^{\prime}}{\rho_{0}}$. Substituting expressions (6) into the system (3-5) :

$$
\begin{gathered}
\left(\frac{\partial}{\partial t}+u \frac{\partial}{\partial z}\right)\left(\frac{1}{r} \frac{\partial^{2} \Psi}{\partial r^{2}}-\frac{1}{r^{2}} \frac{\partial \Psi}{\partial r}+\frac{1}{r} \frac{\partial^{2} \Psi}{\partial z^{2}}\right)-4 u \frac{r}{R^{2}} \Delta \Phi=0 \\
\frac{\partial}{\partial t} \Phi+u \frac{\partial \Phi}{\partial z}+c^{2} \tilde{\rho}+F=0 \\
\left(\frac{\partial}{\partial t}+u \frac{\partial}{\partial z}\right) \tilde{\rho}+\Delta \Phi=0 \\
\frac{\partial F}{\partial z}=\frac{\partial u}{\partial r}\left(\frac{\partial \Phi}{\partial r}-\frac{1}{r} \frac{\partial \Psi}{\partial z}\right)+\left(\frac{\partial}{\partial t}+u \frac{\partial}{\partial z}\right) \frac{1}{r} \frac{\partial \Psi}{\partial r}=0
\end{gathered}
$$

We will look for a solution in the form of:

$$
\begin{array}{ll}
\Phi(r, z, t)=\phi(r) e^{\mathrm{i} \frac{\alpha c t+\beta z}{R}}, & \Psi(r, z, t)=\psi(r) e^{\mathrm{i} \frac{\alpha c t+\beta z}{R}} \\
\widetilde{\rho}(r, z, t)=\Omega(r) e^{\mathrm{i} \frac{\alpha c t+\beta z}{R}}, & F(r, z, t)=f(r) e^{\mathrm{i} \frac{\alpha c t+\beta z}{R}}
\end{array}
$$

Let us introduce the operators:

$$
A=\frac{\partial^{2}}{\partial r^{2}}+\frac{1}{r} \frac{\partial}{\partial r}, \quad B=\frac{\partial^{2}}{\partial r^{2}}-\frac{1}{r} \frac{\partial}{\partial r}
$$


Substituting the form of the solution (11) into equations (7 - 10) and after a series of transformations, we obtain equations for the radial dependences of the acoustic potential and current function:

$$
\begin{gathered}
\left(4 \beta r^{2} \alpha+8 \beta^{2} r^{2} M\left(1-r^{2}\right)+16 M r^{3} \frac{\partial}{\partial r}\right) \phi(r)+ \\
+i \beta\left(B-\beta^{2}\right) \psi(r)-4 \mathrm{i} \alpha r M \frac{\partial}{\partial r} \psi(r)=0 ; \\
\mathrm{i}\left(\alpha+\beta M\left(1-r^{2}\right)\right)\left(B-\beta^{2}\right) \psi(r)-4 r^{2}\left(A-\beta^{2}\right) \phi(r)=0 .
\end{gathered}
$$

We will look for a solution in the linear approximation of the Mach number, in the form of

$$
\phi=\phi_{0}+M \phi_{1}, \quad \psi=\psi_{0}+M \psi_{1}, \quad \beta=\beta_{0}+M \beta_{1},
$$

Substituting the expression (14) into equation (13) we group the components of the equations by powers of $M$. We neglect the second order of smallness and divide the system into two: zero approximation and the first.

Get the equation separately for $\psi_{0}$ and $\phi_{0}$ :

$$
\begin{gathered}
\left(A+\alpha^{2}-\beta_{0}^{2}\right) \phi_{0}=0 \\
\mathrm{i} \alpha\left(B-\beta_{0}^{2}\right) \psi_{0}=-4 r^{2} \alpha^{2} \phi_{0}
\end{gathered}
$$

Then we obtain the equations for the first approximation. The equation for determining $\phi_{1}$ :

$$
\left(A-\beta_{0}^{2}+\alpha^{2}\right) \phi_{1}=\left(-4 \alpha\left(1-r^{2}\right) \beta_{0}+2 \beta_{1} \beta_{0}-4 r \alpha \frac{1}{\beta_{0}} \frac{\partial}{\partial r}\right) \phi_{0}+\frac{\mathrm{i} \alpha^{2}}{\beta_{0} r} \frac{\partial}{\partial r} \psi_{0}
$$

and the equation to determine $\psi_{1}$ :

$$
\begin{gathered}
\left(B-\beta_{0}^{2}\right) \psi_{1}=\left(8 \mathrm{i} r^{2}\left(1-r^{2}\right) \beta_{0}+16 \mathrm{i} r^{3} \frac{1}{\beta_{0}} \frac{\partial}{\partial r}\right) \phi_{0}+ \\
+4 \mathrm{i} r^{2} \alpha \phi_{1}+\left(2 \beta_{1} \beta_{0}+4 \alpha r \frac{1}{\beta_{0}} \frac{\partial}{\partial r}\right) \psi_{0} .
\end{gathered}
$$

We got equations for determining all the components. We solve them.

\subsection{Zero approximation solution}

We have to start the solution for the zero approximation with Eq. (15). The boundary conditions (2) must be taken into account. This problem is a case of acoustic waves propagation in a cylindrical waveguide in the presence of a harmonic oscillations circular source in the absence of a flow. The solution of this problem is presented in [4] if the flow rate is set to zero. It is the sum of the harmonic components of $z$ - normal modes propagating in two directions from the source. In our notation we may write

$$
\varphi^{ \pm}(r, z)=\sum_{n=0}^{\infty} \mp A_{n} J_{0}\left(\mu_{n} r\right) e^{\mp i s_{n} z}, \text { where } A_{n}=\frac{2 \mathrm{i} R v_{0} \sin \left(s_{n} \frac{h}{R}\right)}{J_{0}\left(\mu_{n}\right) s_{n}^{2}}
$$

here the upper sign applies when $z>0$, lower at $z<0, \beta_{0}=\mp s_{n}=\mp \sqrt{\alpha^{2}-\mu_{n}^{2}}, \alpha=\frac{\omega R}{c}, J_{0}$ is the Bessel function of the first kind, of zero order, $\mu_{n},(n=0,1,2 \ldots)$ are zeros of the first kind, first order Bessel functions $\left(J_{1}\right)$. 
In the future we will consider separately $n$ - th mode. Then it is clear that $\phi_{0}(r)=$ $\left(\mp A_{n}\right) J_{0}\left(\mu_{n} r\right)$.

We obtain an expression for the radial component of the current function in the zero approximation of $\psi_{0}(r)$. We substitute this expression (19) for $\phi_{0}(r)$ and $\beta_{0}$ for $n$-th mode in the right part of Eq. (16). Then we get

$$
\left(\frac{\partial^{2}}{\partial r^{2}}-\frac{1}{r} \frac{\partial}{\partial r}-\beta_{0}^{2}\right) \psi_{0}=4 \mathrm{i} \alpha\left(\mp A_{n}\right) r^{2} J_{0}\left(\mu_{n} r\right)
$$

We obtain the solution of the Eq. (20) with zero boundary conditions on the waveguide wall, where the source was taken into account in the calculation (19):

$$
\begin{gathered}
\psi_{0}(r)=-\frac{4 \mathrm{i} \alpha\left(\mp A_{n}\right)}{\alpha^{2}} r^{2} J_{0}\left(\mu_{n} r\right)+\frac{8 \mathrm{i} \alpha\left(\mp A_{n}\right) \mu_{n}}{\alpha^{4}} r J_{1}\left(\mu_{n} r\right)+ \\
+\left(\mp A_{n}\right) \frac{4 \mathrm{i}}{\alpha} \frac{J_{0}\left(\mu_{n}\right)}{J_{1}\left(i \beta_{0}\right)} r J_{1}\left(\mathrm{i} \beta_{0} r\right) .
\end{gathered}
$$

\subsection{Solution in the first approximation}

We find the equations for the first approximation by collecting first-order summands of $M$. We get the equation for $\phi_{1}(r)$ by substituting solutions (19) and (21) into the Eq. (17). The solution of this equation is:

$$
\begin{aligned}
\phi_{1}(r) & =\left(\mp A_{n}\right) J_{1}\left(\mu_{n} r\right)\left(r \frac{8 \beta_{0} / \alpha-4 \alpha \beta_{0} / 3}{2 \mu_{0}}-r \frac{4 \alpha \beta_{0}}{3 \mu_{n}^{3}}+\frac{2 \beta_{0} r^{3}}{3 \mu_{n}}\right)+ \\
& +\left(\mp A_{n}\right) \frac{2 \alpha \beta_{0} r^{2} J_{0}\left(\mu_{n} r\right)}{3 \mu_{n}^{2}}-\frac{\mp 4 \mathrm{i} \alpha A_{n} J_{0}\left(\mu_{n}\right)}{\alpha^{2} J_{1}\left(\mathrm{i} \sqrt{\alpha^{2}-\mu_{n}^{2}}\right)} J_{0}\left(\mathrm{i} \beta_{0} r\right)
\end{aligned}
$$

Upon obtaining this solution, it was shown that zero boundary conditions are satisfied for this function when:

$$
\beta_{1}=\frac{4 \alpha}{3}
$$

Consider the relationship between the acoustic potential phase and the average airflow velocity. It is defined by expression (22). It can be shown that the phase difference of the acoustic signals propagating along and against the flow at a distance of $l$ from the source will be equal:

$$
\Delta \phi_{\text {lam }}=2 \beta_{1} l M=\frac{8}{3} \frac{\omega}{c^{2}} \sqrt{u}
$$

In [1], we obtain a similar expression as Eq. (22) for the phase difference depending on the velocity of the homogeneous flow (with a rectangular plot). From it in a linear approximation of $M$ it can be obtained:

$$
\Delta \phi_{\text {turb }}=2 \frac{\omega}{c^{2}} l \bar{u}
$$

Comparison of expressions (23) and (24) allows to estimate influence of inhomogeneity of a stream on indications of the device measuring speed of a flow by an acoustic phase method. It can be seen that the proportionality factor between the measured velocity and the phase difference in the laminar flow will be $3 / 4$, if we talk about the average cross-section velocity and $3 / 2$, if about the maximum. 


\section{Conclusion}

We considered the problem of acoustic wave propagation in a cylindrical waveguide in an inhomogeneous flow with a parabolic velocity plot. The solution was obtained for the acoustic potential and current function. The amplitude and phase of acoustic waves were expressed in a linear Mach-number representation. This approximation holds because the laminar flow (flow with parabolic plot) is set at low flow rates. Expression (22) makes it possible to determine the influence of the shape of the velocity plot on the phase of the acoustic signal, which is an informative parameter in the phase acoustic method for measuring flow velocities.

This solution can be used to describe the acoustic principle of measuring air flow and velocity, as well as to calibrate the acoustic spirometer by flow.

The work was supported by the Russian Science Foundation, use project No 14-19-01633.

\section{References}

[1] S.Z. Shkundin, V. B. Lashin Metrology. 7 39-43 (1990) (in Russian)

[2] D. D. Landau, E. M. Lifshits Hydrodynamics (Nauka, Moscow, 1988) 733 (in Russian)

[3] L. G. Loytzyansky Mechanics of liquid and gas (Drofa, Moscow, 2003) 840 (in Russian)

[4] A.D. Lapin Akustiko-aehrodinamicheskie issledovaniya: sbornik. pod red. RimskogoKorsakova.[ Acoustic-aerodynamic studies: a collection. ed. by Rimsky-Korsakov] (Moscow, 1975 ) 57-60 (in Russian)

[5] N.I. Sidnyaev, N.M. Gordeeva, Journal of Applied and Industrial Mathematics 9, Issue $1,110-118$ (2015)

[6] I.K. Marchevskii, V.V. Puzikova, Journal of Machinery Manufacture and Reliability 46, Issue 2, 114-119 (2017)

[7] A.A. Pozhalostin, D.A. Goncharov, Russian Aeronautics 58, Issue $137-41$ (2015)

[8] E.V. Murashkin, Y. N. Radayev J. Phys.: Conf. Ser. 937012031 (2017)

[9] A.Yu. Varaksin High Temperature 53, No. 3 423-444. (2015) 